\title{
The natural history of 21-hydroxylase autoantibodies in autoimmune Addison's disease
}

\section{Anette Boe Wolff(1) 1,2,3, Lars Breivik 10,2,3, Karl Ove Hufthammer ${ }^{4}$, Marianne Aardal Grytaas ${ }^{2,3}$, EirikBratland ${ }^{1,2,5}$, Eystein Sverre Husebye ${ }^{1,2,3}$ and Bergithe Eikeland Oftedal(i) 1,2}

${ }^{1}$ Department of Clinical Science, ${ }^{2}$ K.G. Jebsen Center for autoimmune disorders, University of Bergen, Norway, ${ }^{3}$ Department of Medicine, ${ }^{4}$ Centre for Clinical Research, and ${ }^{5}$ Department of Medical Genetics, Haukeland University Hospital, Bergen, Norway
Correspondence should be addressed to E S Husebye Email eystein.sverre.husebye@ helse-bergen.no

\section{Abstract}

Background: The most common cause of primary adrenal failure (Addison's disease) in the Western world is autoimmunity characterized by autoantibodies against the steroidogenic enzyme 21-hydroxylase (CYP21A2, 21OH). Detection of $21 \mathrm{OH}$-autoantibodies is currently used for aetiological diagnosis, but how levels of $21 \mathrm{OH}$-autoantibodies vary over time is not known.

Setting: Samples from the national Norwegian Addison's Registry and Biobank established in $1996(n=711)$. Multiparameter modelling of the course of $210 \mathrm{H}$-autoantibody indices over time.

Results: $210 \mathrm{H}$-autoantibody positivity is remarkably stable, and $>90 \%$ of the patients are still positive 30 years after diagnosis. Even though the antibody levels decline with disease duration, it is only rarely that this downturn reaches negativity. $210 \mathrm{H}$-autoantibody indices are affected by age at diagnosis, sex, type of Addison's disease (isolated vs autoimmune polyendocrine syndrome type I or II) and HLA genotype.

Conclusion: $210 \mathrm{H}$-autoantibodies are reliable and robust markers for autoimmune Addison's disease, linked to HLA risk genotype. However, a negative test in patients with long disease duration does not exclude autoimmune aetiology.

\section{Introduction}

Acquired primary adrenal insufficiency (PAI) has multiple causes including autoimmunity, infections such as tuberculosis, genetic disorders, haemorrhage, and surgical removal. Sometimes the reason is obvious (surgery); in other cases, additional work-up is needed to ascertain the origin. Autoimmunity accounts for 75-96\% of the cases in industrialised countries $(1,2,3)$, defined by the presence of 21-hydroxylase autoantibodies (21OH-Abs) $(4,5,6,7,8)$. Additional clues are the presence of organ-specific autoimmune comorbidities such as hypothyroidism, type 1 diabetes mellitus, and vitiligo.
$21 \mathrm{OH}-\mathrm{Abs}$ are present in $80-90 \%$ of patients with PAI in cross-sectional studies when known non-autoimmune causes have been excluded $(9,10)$, and is used in the diagnostic work up for this disorder (11). Data from a limited number of patients indicate that the frequency is higher shortly after diagnosis (>95\%) (12), while it tends to fall with increasing disease duration reaching about 50\% after 20 years $(7,12)$. Thus, if $210 \mathrm{H}$-Abs are assayed many years after diagnosis, a negative result does not exclude autoimmunity. Conversely, $21 \mathrm{OH}$-Abs can be present in individuals with completely normal adrenal function, where their presence signals a future risk of developing

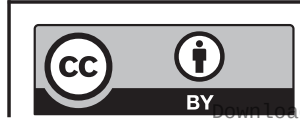

This work is licensed under a Creative Commons Attribution 4.0 International License. 
overt PAI $(13,14)$. Both environmental and genetic factors are involved in the pathogenesis, and certain HLA alleles confer a high risk of developing the disease $(15,16,17,18)$.

We have limited information on the natural history of 21OH-Abs in Addison's disease and how many become autoantibody negative over time. We hypothesise that the diagnostic value of $21 \mathrm{OH}$-Abs declines with time and that patients might have autoimmune Addison's disease despite the lack of autoantibodies. By using a national registry and biobank established in 1996 with serial samples including the majority of Norwegian patients with Addison's disease, we aimed to investigate the robustness of the $21 \mathrm{OH}-\mathrm{Abs}$ assay and its clinical value in defining autoimmune PAI.

\section{Methods}

\section{Patients}

The study was approved by the Regional Committees for Medical and Health Research Ethics (project nos. 2013/1504 and 2018/1417), with written informed consent obtained from each participant after full explanation of the purpose and nature of all procedures used. The National Norwegian Addison Registry (ROAS) collates clinical information, demographics, and biological samples from patients with PAI. 21OH-Abs status is assayed in all patient samples when they are included in the registry and every time a successive sample is included in the biobank. The samples are analysed with the same $21 \mathrm{OH}-\mathrm{Abs}$ assay method in the same laboratory. Those with a positive result are classified as autoimmune PAI. Presence of autoimmune polyendocrine syndrome type I (APS-I) is defined by two of the following three components PAI, hypoparathyroidism, and chronic mucocutaneous candidiasis, type I interferon antibodies and disease-causing variants in the Autoimmune Regulator (AIRE) gene. All samples in the registry are screened for interferon omega antibodies to exclude undiagnosed APSI. APS-II is categorised as PAI concomitantly with type 1 diabetes and/or autoimmune thyroid disease. Details regarding clinical criteria for PAI and comorbidities are given in previous publications on the Norwegian PAI cohort and registry (20).

Patients in ROAS without $210 \mathrm{H}-\mathrm{Abs}$ are screened for other causes, usually with imaging of the adrenals in adult patients (to reveal signs of infection, tumour and haemorrhage), and genetic screening for causes such as adrenal hypoplasia congenita (DAX1-mutations) (21) and adrenoleukodystrophy (accumulation of very-long chain fatty acids and mutations in ABCD1) (22). Those without known cause are classified as idiopathic, but probably autoimmune PAI. Altogether 711 patients with autoimmune and idiopathic PAI were included, and their characteristics are reported in Table 1. Exclusion criteria were PAI-patients with known non-autoimmune causes.

In addition, we re-assayed $21 \mathrm{OH}$-Abs in serial samples (total number of samples, 389, range 3-15 samples from each patient, median 9) from 45 of the 711 patients. This 'verification cohort' included patients who were recruited to the registry between 1996 and 2001 with a minimum of three samples spanning at least 15 years and with at least one sample positive for $21 \mathrm{OH}$-Abs. Samples from a particular patient were analysed on the same plate to avoid plate-to-plate fluctuations in indices of $21 \mathrm{OH}$-Abs. Patients with APS-I verified by sequencing of AIRE (23) and diagnosed with PAI were included in the registrybased segment of the study (see below). A flow chart on the included patients and samples in this study is shown in Fig. 1.

\section{Assay of $210 \mathrm{H}-\mathrm{Abs}$}

An in-house radio-ligand binding assay was employed to detect $21 \mathrm{OH}$-Abs as described previously (24). A positive (index 1000) and negative (index 0) control was used to calculate the $21 \mathrm{OH}-\mathrm{Abs}$ index. The threshold for

Table 1 Characteristics of the 711 included patients with autoimmune Addison's disease. Data are presented as mean \pm S.D.

\begin{tabular}{|c|c|c|c|}
\hline & PAI & APS-II & APS-I with PAl* \\
\hline Patients, $n$ & 305 & 372 & 34 \\
\hline Frequency of females (\%) & 50.3 & 72.0 & 41.2 \\
\hline Age at diagnosis (years) & $33.1 \pm 15.4$ & $36.6 \pm 14.8$ & $15.7 \pm 9.6$ \\
\hline Time between diagnosis and first sample (years) & $11.5 \pm 13.5$ & $11.2 \pm 12.7$ & $15.0 \pm 15.3$ \\
\hline $210 \mathrm{H}-\mathrm{Abs}$ index & $577 \pm 356$ & $620 \pm 319$ & $317 \pm 228$ \\
\hline Positivity for $210 \mathrm{H}-\mathrm{Abs}(\%)$ & 88.2 & 93.8 & 85.3 \\
\hline Males & $89.4 \%$ & $93.2 \%$ & \\
\hline Females & $87.6 \%$ & $93.6 \%$ & \\
\hline
\end{tabular}




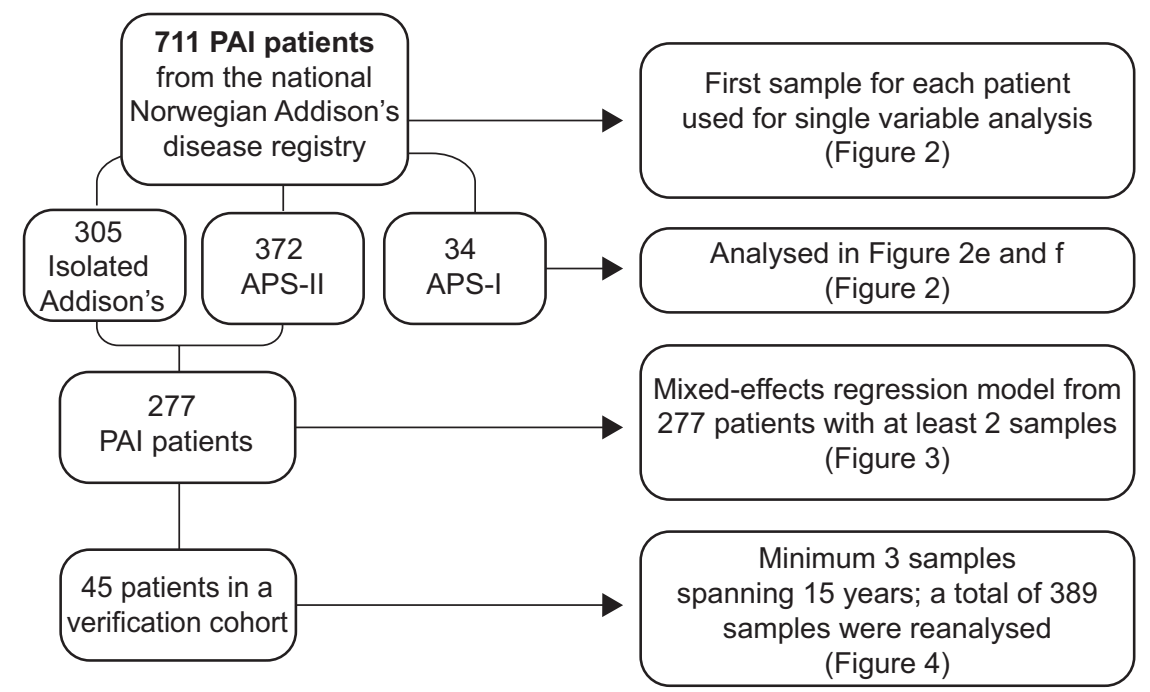

\section{Figure 1}

Flow chart describing the patients in this study. positivity has been set by assaying 150 healthy controls and calculating the mean $21 \mathrm{OH}-\mathrm{Abs}$ index +3 s.D. This assay, including the threshold for positivity and variance numbers, has further been tested and verified by comparing with other European laboratories in the Euradrenal Consortium (10). The samples are usually analysed within a month of sampling. All samples are stored at $-80^{\circ} \mathrm{C}$.

\section{Human leukocyte antigen (HLA) determination and risk of developing autoimmune PAl}

Genotypes for HLA-DRB1 and HLA-DQB1 were analysed with a PCR-based sequence-specific oligonucleotide probe system at four-digit resolution or imputed from the Global Screening Array chip $(15,18)$. The HLA-DQA1 alleles and the HLA-DRB1-DQA1-DQB1 haplotypes were deduced based on known patterns of linkage disequilibrium in the Norwegian population. The HLA-DRB1-DQA1-DQB1 genotypes were stratified into three risk categories according to previously reported risk HLA variants for autoimmune
PAI $(12,15)$ (Table 2), also supported by data from a recent genome-wide association study on autoimmune PAI (18).

HLA-data from 285 of the included PAI patients (isolated PAI and APS-II) with disease duration $<5$ years at sampling were available. The genotypes were categorised into the three groups according to the estimated risk these variants confer for developing PAI ; $n=70$, low risk, $n=115$, intermediate risk, and $n=100$, high risk (Table 2 ). In the mixed effects model explained below where all patients in the registry with $>2$ samples were included, there were 80, 91 and 107 individuals in each of the risk groups, respectively.

\section{Statistical analysis}

Impact on individual parameters for $210 \mathrm{H}$-Abs frequency and index

Statistical tests on frequencies of groups were performed with logistic regression analysis (Fig. 2A and B). A parametric Student's $t$-test was employed to compare sex-

Table 2 Classification of HLA genotypes and applied risk class.

\begin{tabular}{|c|c|}
\hline Risk class (this study) & Allele 1 (HLA-DRB1-DQA1-DQB1) \\
\hline 1 & *0301-*0501-*0201 \\
\hline 1 & *0404-*0301-*0302 \\
\hline 1 & *0301-*0501-*0201 \\
\hline 2 & *0301-*0501-*0201 \\
\hline 3 & *0404-*0301-*0302 \\
\hline 3 & Intermediate* \\
\hline 3 & Intermediate* \\
\hline 3 & Low* \\
\hline
\end{tabular}

\begin{tabular}{l} 
Allele 2 (HLA-DRB1-DQA1-DQB1) \\
\hline *0404-*0301-*0302 \\
*0404-*0301-*0302 \\
*0301-*0501-*0201 \\
Any other DRB1 allele \\
Any other DRB1 allele \\
Intermediate * \\
Low* \\
Low*
\end{tabular}

\begin{tabular}{l} 
RISK category \\
\hline Very high \\
High \\
High \\
Intermediate \\
Low \\
Low \\
Very low \\
Very low
\end{tabular}

* Intermediate: The combination of any of the following HLA-DRB1-DQA1-DQB1 haplotypes: *1401-*0101-*0503, *15-*0102-*0602/*0611, *07-*0201*0303, *0401-*0301-*0301, *0801-*0401-*04, *11-*0501 -*0301 and/or *12-*0501-*0301.

*Low risk: Combination of the HLA-types in the 'Intermediate risk' category or the combination of any of the following HLA-DRB1-DQA1-DQB1

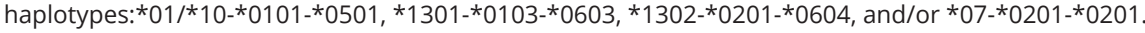


differences regarding 21OH-Abs indices, and a Pearson's chi test to compare 21OH-Abs frequencies (Fig. 2C). ANOVA was used for comparing multiple groups, and Tukey's multiple comparisons test was applied to compare statistical differences between groups (Fig. 2D).

\section{Mixed effects regression model}

To fully exploit our longitudinal sampling of patients with autoimmune PAI, and because autoantibodies against $21 \mathrm{OH}$ are assayed in every sample recruited to ROAS, we included all samples from PAI patients with at least two measurements, excluding APS-I $(n=711)$ to generate a mixed-effects regression model of the $21 \mathrm{OH}$-Abs course over time. We adjusted for disease duration (sampling date from date of diagnosis), age, sex, type of PAI (isolated Addison's disease vs APS-II), and HLA-risk genotype (low risk, intermediate risk, high risk) as covariates.

To model the trajectories of $21 \mathrm{OH}-\mathrm{Abs}$ over time, we fitted a mixed-effects regression model. The explanatory variables were disease duration (years from diagnosis), age at diagnosis, sex, HLA risk category (three levels), and APS type (isolated Addison's disease vs APS-II). Patients with APS-I were excluded in this model due to low numbers and monogenic cause.

Initial visualisations of the data indicated a non-linear association with years from diagnosis, so this variable was included as second-degree orthogonal polynomial. Random effects were included for the coefficients of the polynomial (including the intercept). This takes into account the dependence between samples from the same individual, and it allows each patient to have their own second-degree curve. However, unlike models where one fits second-degree curves for each patient separately, when estimating the curves in the mixed-effect model, information is 'borrowed' from the whole population. This allowed us to reliably estimate second-degrees curves also for patients with very few samples. For this model, we included data from all patients who had at least two available samples $(n=227)$.

High 21OH-Abs indices seemed to have larger variance. In the regression model, we therefore modelled the variance as a linear function of the predicted values. Examination of residuals plots indicated that the resulting model fitted the data well.

The data was analysed with Graphpad Prism 7 and $\mathrm{R}$ version 4.0 .2 (25). 95\% confidence intervals were calculated with a fraction of total analysis using the Wilson/Brown method. The mixed-effects model was fitted with the $\mathrm{R}$
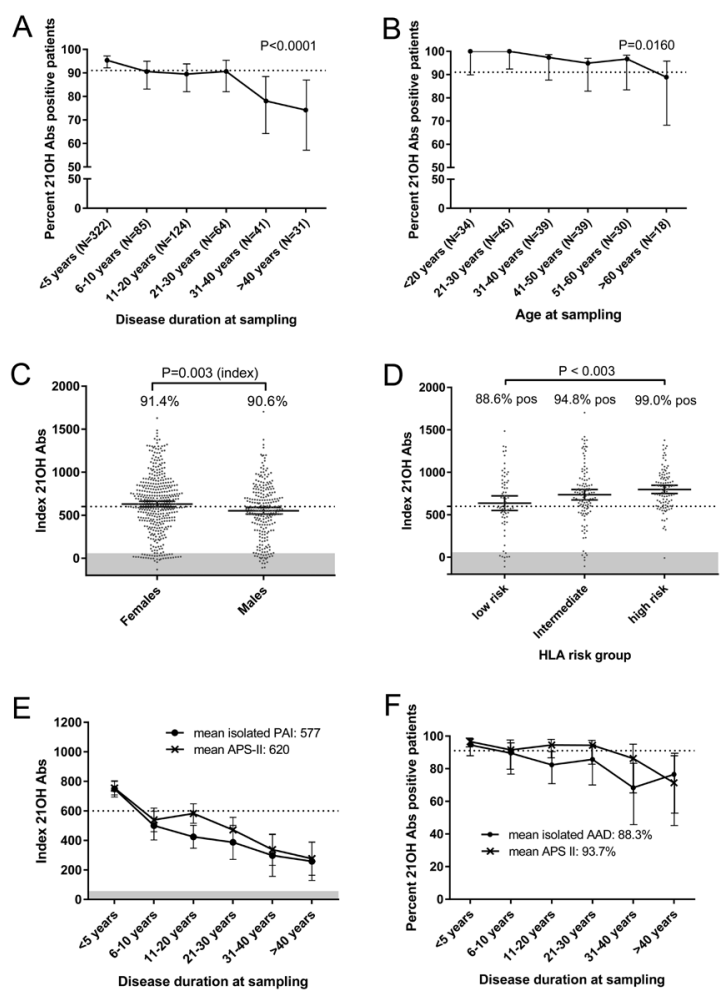

\section{Figure 2}

Individual variables' impact on $210 \mathrm{H}$-Abs frequency and indices (A) Percentage of isolated PAI and APS-II patients positive for antibodies against $210 \mathrm{H}(21 \mathrm{OH}-\mathrm{Abs})$ separated in groups according to disease duration at sampling. The statistical test was done with logistic regression analysis. (B) Percentage of isolated PAI and APS-II patients positive for $210 \mathrm{H}$-Abs sampled within 1 year of diagnosis in relation to age at diagnosis. Statistical analysis was done with logistic regression analysis. (C) $210 \mathrm{H}$-Abs-indicies in males and females. Percentage positive for each sex is shown inside the corresponding box. Statistical test for indices between the groups was achieved by a parametric t-test while statistical differences between the frequencies were done by a Pearson's chi test. (D) $210 \mathrm{H}$-Abs indices stratified by HLA-risk groups given in Table 2 and percentage positives in each group. Statistical differences were calculated by ANOVA and Tukey's test. (E) $210 \mathrm{H}$-Abs-indices stratified into phenotypic groups and disease duration at sampling; isolated PAI (black) and APS-II (red). (F) Percent positive for $21 \mathrm{OH}$-Abs stratified to disease duration at sampling; isolated PAI (black) and APS-II (red). The dashed line (A, B and F) represents the mean positivity for autoantibodies against $210 \mathrm{H}-\mathrm{Abs}$ in the whole isolated, AAD autoimmune PAI and APS-II cohort. The dashed line ( $C$ and $E$ ) represents the mean indices of $210 \mathrm{H}-\mathrm{Abs}$. The shaded area (C-E) in the lower part of the graph represent where the test gives negative results (sets the threshold for positivity). $95 \% \mathrm{Cl}$ is shown by vertical bars. 
package 'nlme' version 3.1-149 (26). $P$ values $\leq 0.05$ were characterised as statistically significant (Table 3 ).

\section{Results}

\section{Description of the patient cohort}

Altogether 711 patients were included, 305 with isolated PAI, 34 with APS-I, and 372 with APS-II. The sex ratio was equal among isolated PAI (50.3\% females), and APS-I (41.2\% females), while females dominated the APS-II group (72.0\%). Mean age at diagnosis was 33.1 (s.D. 15.4), 15.7 (s.D. 9.6) and 36.6 (s.D. 14.8) years for isolated PAI, APS-I and APS-II, respectively. The mean time between diagnosis and the first available blood sample varied between 11.2 and 15.7 years for the disease groups. Positivity of $21 \mathrm{OH}-\mathrm{Abs}$ was $>85 \%$ in all three groups; the mean 21OH-Abs indices were higher for isolated PAI (mean: 577) and APS-II (620), than for APS-I (317). The results are summarised in Table 1.

\section{H-Abs in the first available sample according to age, disease duration, sex, HLA risk and Addison's disease type}

Intriguingly, $>90 \%$ of PAI-patients with diagnosis up to at least 60 years of age had $21 \mathrm{OH}-\mathrm{Abs}$, and patients retained these autoantibodies up to 30 years after diagnosis (Fig. 2A and $\mathrm{B}$ ). For patients with disease duration that exceeds 30 years, the $21 \mathrm{OH}-\mathrm{Abs}$ frequency fell to $\sim 75 \%$ in this 'onevariable'-analysis (Fig. 2A, $P<0.0001$ ), showing a trend towards a decrease of $21 \mathrm{OH}$ levels over time.

We further investigated whether sex, HLA genotype, and type of PAI influenced the $21 \mathrm{OH}$-Abs status as single

Table 3 Multivariable mixed-effects longitudinal regression model for $210 \mathrm{H}-\mathrm{Abs}(n=1287$ observations from 277 individuals).

\begin{tabular}{|c|c|c|c|}
\hline Variable & Coeff. & $95 \% \mathrm{Cl}$ & P-value \\
\hline Time from diagnosis (nonlinear) & - & - & $<0.001$ \\
\hline Age at diagnosis (years) & -2.2 & -4.2 to -0.3 & 0.03 \\
\hline Sex, female & 117 & 55 to 178 & $<0.001$ \\
\hline \multicolumn{4}{|l|}{ HLA risk group } \\
\hline Low & 0 & - & - \\
\hline Intermediate & 99 & 32 to 167 & 0.004 \\
\hline High & 69 & 19 to 119 & 0.007 \\
\hline APS-II, yes & 75 & 16 to 124 & 0.01 \\
\hline
\end{tabular}

variables. To this end, we categorised the $21 \mathrm{OH}-\mathrm{Abs}$ indices from the first sample from each patient according to presence of (i) an autoimmune syndrome or not (isolated PAI, APS-I, and APS-II), (ii) sex, and (iii) HLA risk group, looking at one parameter at a time. For HLA, only patients with disease duration from diagnosis to sampling $<5$ years were included. While there was a difference in indices of $21 \mathrm{OH}-\mathrm{Abs}$ between females and males $(P=0.003$, 95\% CI $(26.1$ to 130$))$, no difference in $21 \mathrm{OH}-\mathrm{Abs}$ frequencies between females $(91.4 \%)$ and males (90.6\%) was detected (Fig. 2C). Intriguingly, we found that presence of $21 \mathrm{OH}-\mathrm{Abs}$ correlates with HLArisk genotype. In patients with low-risk HLA-types, $88.6 \%$ had $210 H-A b s$ (mean index 637), as opposed to $94.8 \%$ in the intermediate group (mean index 736) and $99.0 \%$ in the high-risk group (mean index 798) (overall ANOVA $P<0.00002$ ) (Fig. 2D).

Looking at the three patient categories of (i) isolated PAI, (ii) APS-I and (iii) APS-II, we discovered several interesting patterns (Fig. 2E and F) (APS-I not shown). First, there was a trend that patients with APS-I had lower levels of 21OH-Abs until 31-40 years, but then indices increased compared to patients with isolated PAI. The pattern of $21 \mathrm{OH}$-Abs levels in isolated PAI and APS-II were similar at very young ages ( $<10$ years), starting at indices about 700-800 with approximately 95\% of samples positive. At older disease durations, APS-II patients tended to have both higher frequencies and indices of $210 \mathrm{H}-\mathrm{Abs}$ than the isolated, autoimmune PAI group. Both groups had declining frequencies and indices over time, although indices were still positive in the area of 200-300 and at frequencies of $70-80 \%$, 40 years after diagnosis.

\section{Unified model of $210 H-A b s$ indices shows a decline with disease duration, and dependence on sex, type of Addison's disease and HLA-risk group}

The unified model shows that indeed the $210 \mathrm{H}-\mathrm{Abs}$ indices contract over time, but it seldom reaches values below the threshold for positivity, at least not in those with intermediate and high risk HLA genotypes (Fig. 3). The modelled indices are higher in females than males throughout the time span in all HLA categories. Looking at HLA risk groups, the $21 \mathrm{OH}$-Abs is constantly lower in the low risk group compared to intermediate and highrisk group (Fig. 3). The strongest association to $21 \mathrm{OH}-\mathrm{Abs}$ was disease duration, followed by HLA risk group, and then sex (Table 3).

To verify the registry results of declining levels of $21 \mathrm{OH}-\mathrm{Abs}$ due to long disease duration (Figs 2A and 3), 


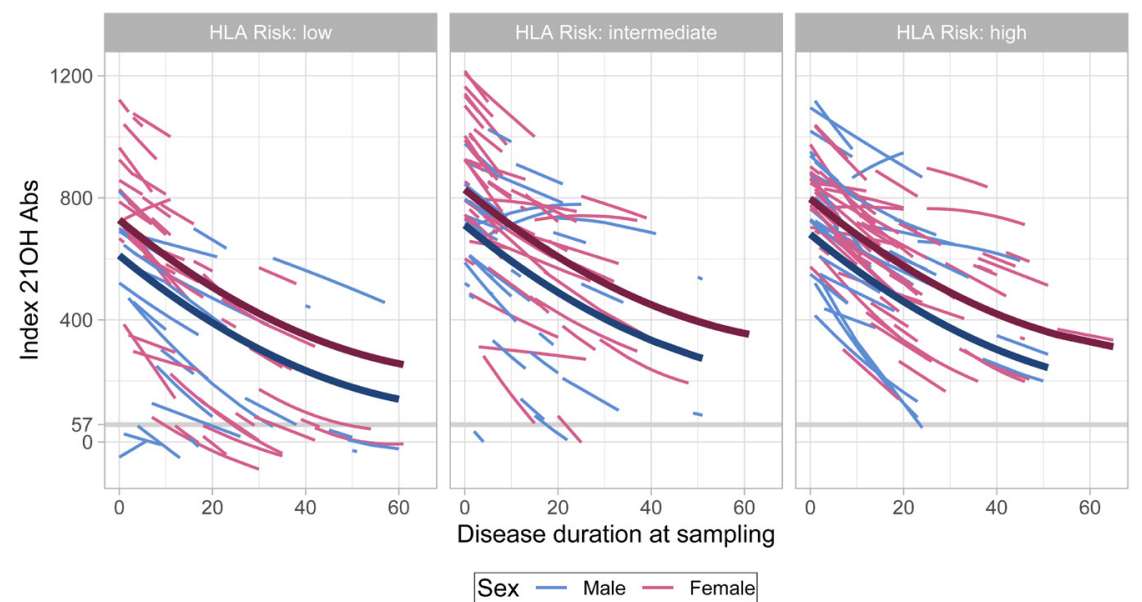

\section{Figure 3}

Estimated individual trajectories from mixed-effects longitudinal regression model for $210 \mathrm{H}$-Abs $(n=1287$ observations from 277 individuals). The lines corresponds to individual patients. Includes data from patients with at least two samples. The thick lines show the predicted trajectory for a 'typical' patient (all random effects set to 0 ), that is a 40-year-old female with APS-II. we reinvestigated longitudinal samples in 45 patients, running a total of 389 samples from the same patients in the same assay to exclude inter-assay variation (Fig. 4). We found that some patients had antibody levels that decreased very early after diagnosis, sometimes becoming negative. Others were stable at a high level over an extended period of time, while yet others revealed a pattern with large fluctuations in indices. Importantly, the curves have similar patterns for each patient when comparing data from the registry and the verification study (Fig. 4 and Supplementary Fig. 2, see section on supplementary materials given at the end of this article). There are, however, some deviations between the two analysis points, resulting in a fan-shaped deviation graph (Supplementary Fig. 1). This shows that the analytical variation is larger for higher indices, and lower for the points around the threshold for positivity. The stability of the assay, the independence of the person performing the assay and the adequate stable results after repeated freezing and thawing of samples is highlighted by the good correlation between the biobank-recorded value and our verification study.

\section{Discussion}

We have investigated the robustness and clinical value of analyzing autoantibodies against $21 \mathrm{OH}$ in patients with PAI, APS-I, and APS-II and how they persist over the time course of the disease. $21 \mathrm{OH}$-Abs have been analysed in samples from a national registry with over 700 patients, with samples spanning more than 20 years for several of them. The 21OH-Abs have also been analysed in the context of sex, HLA risk genotypes, and underlying genetic mutations (APS-I). In addition, we validated our finding in a subgroup of patients by running longitudinal samples. Our main finding is that the $21 \mathrm{OH}-\mathrm{Abs}$ are remarkable stable, even up to 30 years after diagnosis.

When only considering the first available sample from each patient and looking at each of the factors individually (duration between diagnosis and sampling, age, sex, presence of an APS and HLA risk category), we found that all factors statistically contribute to the autoantibody indices, which declined during disease duration. As APS-I is a rare syndrome, only 34 patients could be included, and the results for this group should be interpreted with caution. Due to their low number and the monogenic form of disease these samples were excluded from the rest of the study. Making a unified model for the course of $21 \mathrm{OH}-\mathrm{Abs}$ using all available samples from the start of ROAS in 1996, we show that all included co-factors affected the $210 \mathrm{OH}-\mathrm{Abs}$ level.

A decline in autoantibody index in relation to age or disease duration could prevent correct diagnosis of autoimmune disorders. However, even though we found such a decline in 21OH-Abs positivity, more than $90 \%$ of the patients still had a positive $210 \mathrm{H}-\mathrm{Abs}$ index 30 years after diagnosis. This is in agreement with a previous study from this cohort of patients (12) and verifies that $21 \mathrm{OH}-\mathrm{Abs}$ are excellent biomarkers for PAI. It also suggests that autoantibodies are generated for decades after the diagnosis is made. Although beyond the scope of this study, it raises the question of which factors are still priming the autoimmune reaction leading to production of these autoantibodies. One possibility is the existence of a small functional adrenal that keeps triggering the response, consistent with our results recent results that about $30 \%$ of patients have some residual adrenal function. from a newly published study showing 


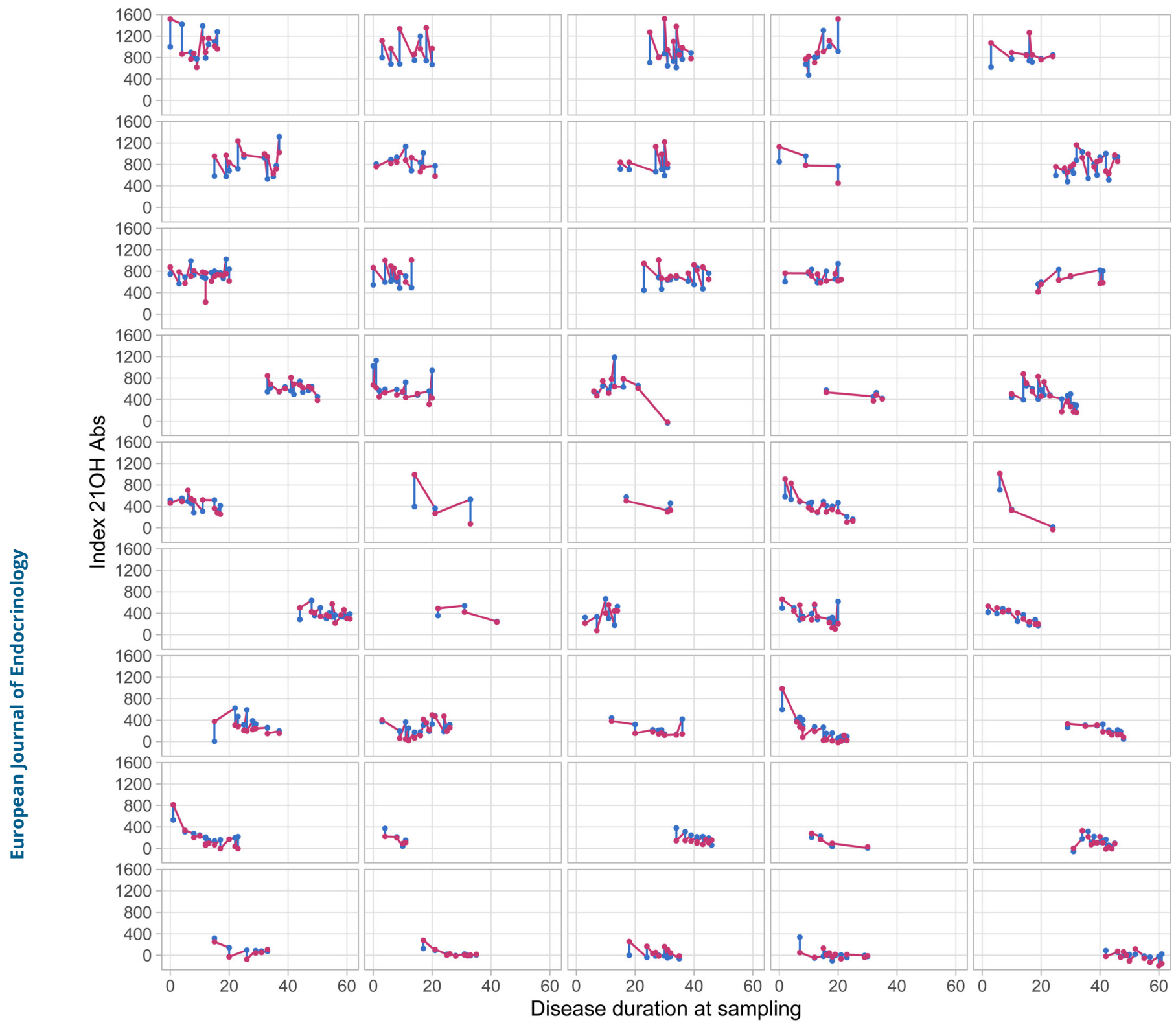

Analysis $\rightarrow$ Original $\rightarrow$ Verification

\section{Figure 4}

Course of $210 \mathrm{H}$-Abs in the 45 patients from the verification study ( $\mathrm{n}=389$ samples) assayed at inclusion in the registry and in the verification study.

presence of adrenal rest, even after decades of clinical disease (27).

We were further intrigued by the differences in $21 \mathrm{OH}-\mathrm{Abs}$ positivity related to HLA, from $88 \%$ in the 'low risk HLA-group' to $>99 \%$ in the 'high HLA risk group'. HLA is an immunological determinant as it is the molecule that presents external or internal peptides to T cells. The HLA genotype works as an individuals' fingerprint which determines what peptides an immune reaction can be mounted against. Indeed, PAI is a highly heritable disease (28), and a large component of the heritability relies on HLA-genotypes $(12,15,16,17$, $29,30,31,32,33)$. Consistent with previous studies, a recent GWAS concluded that the risk was dominated by HLA-DQB1*02:01 (part of the DR3-DQ2 haplotype, $\mathrm{OR}=5.71$ ) and HLA-DQB1*03:02 (part of the DR4-DQ8 
haplotype, OR=5.57) (18). Our findings of less 21OH-Abs in the low-risk HLA group opens for speculation if other hitherto unknown autoantigens are at work. If such autoantibodies exist, they would be valuable additional diagnostic tools and might shed light on the pathogenic actions underlying adrenal autoimmunity.

In conclusion, we have here shown that $21 \mathrm{OH}-\mathrm{Abs}$ positivity is remarkably stable over time, providing a robust biomarker to establish the presence of autoimmune PAI. Even if the autoantibody titre does decrease during long duration time, $>90 \%$ of patients still retain $21 \mathrm{OH}-\mathrm{Abs} 30$ years after diagnosis.

\section{Supplementary materials}

This is linked to the online version of the paper at https://doi.org/10.1530/ EJE-20-1268.

\section{Declaration of interest}

The authors declare that there is no conflict of interest that could be perceived as prejudicing the impartiality of the research reported.

\section{Funding}

The study was funded by the Western Norway Regional Health Authority, the Norwegian Research Council and the K.G. Jebsen Center for autoimmune disorders.

\section{Acknowledgements}

The authors deeply acknowledge the patients and doctors that are involved and included in the ROAS registry and biobank. The authors are indebted to the technicians Elisabeth Halvorsen and Hajirah Muneer, and especially to HUNT laboratory for providing isolated DNA from blood.

\section{References}

1 Husebye ES, Allolio B, Arlt W, Badenhoop K, Bensing S, Betterle C, Falorni A, Gan EH, Hulting AL, Kasperlik-Zaluska A et al. Consensus statement on the diagnosis, treatment and follow-up of patients with primary adrenal insufficiency. Journal of Internal Medicine 2014275 104-115. (https://doi.org/10.1111/joim.12162)

2 Saverino S \& Falorni A. Autoimmune Addison's disease. Best Practice and Research: Clinical Endocrinology and Metabolism 202034101379. (https://doi.org/10.1016/j.beem.2020.101379)

3 Betterle C, Presotto F \& Furmaniak J. Epidemiology, pathogenesis, and diagnosis of Addison's disease in adults. Journal of Endocrinological Investigation 201942 1407-1433. (https://doi. org/10.1007/s40618-019-01079-6)

4 Conrad K, Roggenbuck D, Reinhold D \& Sack U. Autoantibody diagnostics in clinical practice. Autoimmunity Reviews 201211 207-211. (https://doi.org/10.1016/j.autrev.2011.05.014)

5 Erichsen MM, Lovas K, Fougner KJ, Svartberg J, Hauge ER, Bollerslev J, Berg JP, Mella B \& Husebye ES. Normal overall mortality rate in Addison's disease, but young patients are at risk of premature death. European Journal of Endocrinology 2009160 233-237. (https:// doi.org/10.1530/EJE-08-0550)
6 Laureti S, Aubourg P, Calcinaro F, Rocchiccioli F, Casucci G, Angeletti G, Brunetti P, Lernmark A, Santeusanio F \& Falorni A. Etiological diagnosis of primary adrenal insufficiency using an original flowchart of immune and biochemical markers. Journal of Clinical Endocrinology and Metabolism 199883 3163-3168. (https:// doi.org/10.1210/jcem.83.9.5103)

7 Laureti S, De Bellis A, Muccitelli VI, Calcinaro F, Bizzarro A, Rossi R, Bellastella A, Santeusanio F \& Falorni A. Levels of adrenocortical autoantibodies correlate with the degree of adrenal dysfunction in subjects with preclinical Addison's disease. Journal of Clinical Endocrinology and Metabolism 199883 3507-3511. (https://doi. org/10.1210/jcem.83.10.5149)

8 Winqvist O, Gebre-Medhin G, Gustafsson J, Ritzen EM, Lundkvist O, Karlsson FA \& Kampe O. Identification of the main gonadal autoantigens in patients with adrenal insufficiency and associated ovarian failure. Journal of Clinical Endocrinology and Metabolism 199580 1717-1723. (https://doi.org/10.1210/ jcem.80.5.7745025)

9 Husebye ES, Perheentupa J, Rautemaa R \& Kampe O. Clinical manifestations and management of patients with autoimmune polyendocrine syndrome type I. Journal of Internal Medicine 2009265 514-529. (https://doi.org/10.1111/j.1365-2796.2009.02090.x)

10 Falorni A, Bini V, Betterle C, Brozzetti A, Castano L, Fichna M, Kampe O, Mellgren G, Peterson P, Chen S et al. Determination of 21-hydroxylase autoantibodies: inter-laboratory concordance in the Euradrenal International Serum Exchange Program. Clinical Chemistry and Laboratory Medicine 201553 1761-1770. (https://doi. org/10.1515/cclm-2014-1106)

11 Bornstein SR, Allolio B, Arlt W, Barthel A, Don-Wauchope A, Hammer GD, Husebye ES, Merke DP, Murad MH, Stratakis CA et al. Diagnosis and treatment of primary adrenal insufficiency: An endocrine society clinical practice guideline. Journal of Clinical Endocrinology and Metabolism 2016101 364-389. (https://doi. org/10.1210/jc.2015-1710)

12 Myhre AG, Undlien DE, Lovas K, Uhlving S, Nedrebo BG, Fougner KJ, Trovik T, Sorheim JI \& Husebye ES. Autoimmune adrenocortical failure in Norway autoantibodies and human leukocyte antigen class II associations related to clinical features. Journal of Clinical Endocrinology and Metabolism 200287 618-623. (https://doi. org/10.1210/jcem.87.2.8192)

13 Coco G, Dal Pra C, Presotto F, Albergoni MP, Canova C, Pedini B, Zanchetta R, Chen S, Furmaniak J, Rees Smith B et al. Estimated risk for developing autoimmune Addison's disease in patients with adrenal cortex autoantibodies. Journal of Clinical Endocrinology and Metabolism 200691 1637-1645. (https://doi.org/10.1210/jc.20050860)

14 Naletto L, Frigo AC, Ceccato F, Sabbadin C, Scarpa R, Presotto F, Dalla Costa M, Faggian D, Plebani M, Censi S et al. The natural history of autoimmune Addison's disease from the detection of autoantibodies to development of the disease: a long follow-up study on 143 patients. European Journal of Endocrinology 2019180 223-234. (https://doi.org/10.1530/EJE-18-0313)

15 Erichsen MM, Lovas K, Skinningsrud B, Wolff AB, Undlien DE, Svartberg J, Fougner KJ, Berg TJ, Bollerslev J, Mella B et al. Clinical, immunological, and genetic features of autoimmune primary adrenal insufficiency: Observations from a Norwegian registry. Journal of Clinical Endocrinology and Metabolism 200994 4882-4890. (https:// doi.org/10.1210/jc.2009-1368)

16 Eriksson D, Bianchi M, Landegren N, Nordin J, Dalin F, Mathioudaki A, Eriksson GN, Hultin-Rosenberg L, Dahlqvist J, Zetterqvist $\mathrm{H}$ et al. Extended exome sequencing identifies BACH2 as a novel major risk locus for Addison's disease. Journal of Internal Medicine 2016280 595-608. (https://doi.org/10.1111/joim.12569)

17 Skinningsrud B, Lie BA, Lavant E, Carlson JA, Erlich H, Akselsen HE, Gervin K, Wolff AB, Erichsen MM, Lovas K et al. Multiple loci in the HLA complex are associated with Addison's disease. Journal of Clinical 
Endocrinology and Metabolism 201196 E1703-E1708. (https://doi. org/10.1210/jc.2011-0645)

18 Eriksson D, Røyrvik EC, Aranda-Guillén M, Berger AH, Landegren N, Artaza H, Hallgren Å, Grytaas M, Ström S, Bratland E et al. GWAS for autoimmune Addison's disease identifies multiple risk loci and highlights AIRE in disease susceptibility. Nature Communications 2021 12 959. (https://doi: 10.1038/s41467-021-21015-8)

19 Erichsen MM, Lovas K, Skinningsrud B, Wolff AB, Undlien DE, Svartberg J, Fougner KJ, Berg TJ, Bollerslev J, Mella B et al. Clinical, immunological, and genetic features of autoimmune primary adrenal insufficiency: observations from a Norwegian registry. Journal of Clinical Endocrinology and Metabolism 200994 4882-4890. (https:// doi.org/10.1210/jc.2009-1368)

20 Reutens AT, Achermann JC, Ito M, Ito M, Gu WX, Habiby RL, Donohoue PA, Pang S, Hindmarsh PC \& Jameson JL. Clinical and functional effects of mutations in the DAX-1 gene in patients with adrenal hypoplasia congenita. Journal of Clinical Endocrinology and Metabolism 199984 504-511. (https://doi.org/10.1210/ jcem.84.2.5468)

21 Kemp S, Huffnagel IC, Linthorst GE, Wanders RJ \& Engelen M. Adrenoleukodystrophy - neuroendocrine pathogenesis and redefinition of natural history. Nature Reviews: Endocrinology 201612 606-615. (https://doi.org/10.1038/nrendo.2016.90)

22 Bruserud Ø, Oftedal BE, Landegren N, Erichsen MM, Bratland E, Lima K, Jorgensen AP, Myhre AG, Svartberg J, Fougner KJ et al. A longitudinal follow-up of autoimmune polyendocrine syndrome Type 1. Journal of Clinical Endocrinology and Metabolism 2016101 2975-2983. (https://doi.org/10.1210/jc.2016-1821)

23 Ekwall O, Hedstrand H, Grimelius L, Haavik J, Perheentupa J, Gustafsson J, Husebye E, Kampe O \& Rorsman F. Identification of tryptophan hydroxylase as an intestinal autoantigen. Lancet 1998 352 279-283. (https://doi.org/10.1016/S0140-6736(97)11050-9)

24 Team RC. R: A Language and Environment for Statistical Computing. R Foundation for Statistical Computing, 2020.

25 Pinheiro J BD, DebRoy S \& Sarkar D. R Core Team nlme: Linear and nonlinear Mixed effects models. R Package Version 3.1-149, 2000.

26 Saevik ÅB, Akerman AK, Methlie P, Quinkler M, Jorgensen AP, Hoybye C, Debowska AJ, Nedrebo BG, Dahle AL, Carlsen S et al.
Residual corticosteroid production in autoimmune Addison disease. Journal of Clinical Endocrinology and Metabolism 2020105 2430-2441. (https://doi.org/10.1210/clinem/dgaa256)

27 Skov J, Hoijer J, Magnusson PKE, Ludvigsson JF, Kampe O \& Bensing S. Heritability of Addison's disease and prevalence of associated autoimmunity in a cohort of 112,100 Swedish twins. Endocrine 201758 521-527. (https://doi.org/10.1007/s12020-0171441-z)

28 Eisenbarth GS, Wilson PW, Ward F, Buckley C \& Lebovita H. The polyglandular failure syndrome: disease inheritance, HLA type, and immune function. Annals of Internal Medicine 197991 528-533. (https://doi.org/10.7326/0003-4819-91-4-528)

29 Kraus AU, Penna-Martinez M, Shoghi F, Seidl C, Meyer G \& Badenhoop K. HLA-DQB1 position 57 defines susceptibility to isolated and polyglandular autoimmunity in adults: interaction With gender. Journal of Clinical Endocrinology and Metabolism 2019104 1907-1916. (https://doi.org/10.1210/jc.2018-01621)

30 Landegren N, Pourmousa Lindberg M, Skov J, Hallgren Å, Eriksson D, Lisberg Toft-Bertelsen T, MacAulay N, Hagforsen E, RaisanenSokolowski A, Saha H et al. Autoantibodies targeting a collecting duct-specific water channel in tubulointerstitial nephritis. Journal of the American Society of Nephrology 201627 3220-3228. (https://doi. org/10.1681/ASN.2015101126)

31 Landegren N, Sharon D, Freyhult E, Hallgren Å, Eriksson D, Edqvist PH, Bensing S, Wahlberg J, Nelson LM, Gustafsson J et al. Proteome-wide survey of the autoimmune target repertoire in autoimmune polyendocrine syndrome type 1. Scientific Reports 2016 6 20104. (https://doi.org/10.1038/srep20104)

32 Sahoo SK, Zaidi G, Srivastava R, Sarangi AN, Bharti N, Eriksson D, Bensing S, Kampe O, Aggarwal A, Aggarwal R et al. Identification of autoimmune polyendocrine syndrome type 1 in patients with isolated hypoparathyroidism. Clinical Endocrinology 201685 544-550. (https://doi.org/10.1111/cen.13111)

33 Yu L, Brewer KW, Gates S, Wu A, Wang T, Babu SR, Gottlieb PA, Freed BM, Noble J, Erlich HA et al. DRB1*04 and DQ alleles: Expression of 21-hydroxylase autoantibodies and risk of progression to Addison's disease. Journal of Clinical Endocrinology and Metabolism 199984 328-335. (https://doi.org/10.1210/jcem.84.1.5414)

Received 4 November 2020

Revised version received 19 January 2021

Accepted 29 January 2021 